\title{
Correction to: Prior bariatric surgery lowers complication rates following spine surgery in obese patients
}

\author{
Peter G. Passias ${ }^{1,2}$ • Samantha R. Horn ${ }^{1}$ - Dennis Vasquez-Montes ${ }^{1} \cdot$ Nicholas Shepard $^{1} \cdot$ Frank A. Segreto $^{1}$. \\ Cole A. Bortz ${ }^{1}$. Gregory W. Poorman ${ }^{1}$. Cyrus M. Jalai ${ }^{1}$. Charles Wang ${ }^{1} \cdot$ Nicholas Stekas $^{1} \cdot$ Nicholas J. Frangella $^{1}$. \\ Chloe Deflorimonte $^{1}$ - Bassel G. Diebo ${ }^{3}$. Micheal Raad ${ }^{4}$. Shaleen Vira ${ }^{1}$ - Jason A. Horowitz ${ }^{5}$. Daniel M. Sciubba ${ }^{4}$. \\ Hamid Hassanzadeh ${ }^{5} \cdot$ Renaud Lafage $^{6} \cdot$ John Afthinos $^{7} \cdot$ Virginie Lafage $^{6}$
}

Published online: 3 October 2019

(C) Springer-Verlag GmbH Austria, part of Springer Nature 2019

Correction to: Acta Neurochirurgica (2018) 160:2459-2465 https://doi.org/10.1007/s00701-018-3722-6

The AHRQ (Agency for Healthcare Research and Quality) has requested the correction of the result Tables 1, 2 and 3 of this study: All stated numbers below 10 shall be modified to read " $<10$ " instead.

The correct tables are shown below:

The online version of the original article can be found at https://doi.org/ $10.1007 / \mathrm{s} 00701-018-3722-6$

Peter G. Passias

Peter.Passias@nyumc.org

1 Department of Orthopaedic Surgery, NYU Langone Medical Center-Orthopaedic Hospital, New York, NY, USA

2 Departments of Orthopaedic and Neurological Surgery, New York Spine Institute, NYU Medical Center - Orthopaedic Hospital, 301 East 17th St, New York, NY 10003, USA

3 Department of Orthopaedic Surgery, SUNY Downstate Medical Center, Brooklyn, NY, USA

4 Department of Neurosurgery, Johns Hopkins Medical Center, Baltimore, MD, USA

5 Department of Orthopaedic Surgery, University of Virginia Medical Center, Charlottesville, VA, USA

6 Department of Orthopaedic Surgery, Hospital for Special Surgery, New York City, NY, USA

7 Staten Island University Hospital, Staten Island, NY, USA 
Table 1 Complications for all spine surgeries compared between morbidly obese patients with and without a history of bariatric surgery

\begin{tabular}{|c|c|c|c|c|c|}
\hline \multirow[t]{2}{*}{ Complications } & \multicolumn{2}{|c|}{ Bariatric surgery history $(N=1939)$} & \multicolumn{2}{|c|}{ Morbidly obese $(N=1625)$} & \multirow[t]{2}{*}{$P$ value } \\
\hline & $N$ & Percentage & $N$ & Percentage & \\
\hline Bowel issues & 193 & $9.95 \%$ & 207 & $12.74 \%$ & $0.009 *$ \\
\hline Bladder issues & 34 & $1.75 \%$ & 37 & $2.28 \%$ & 0.265 \\
\hline Neurologic & 35 & $1.81 \%$ & 32 & $1.97 \%$ & 0.719 \\
\hline Muscle weakness & $\leq 10$ & $0.15 \%$ & $\leq 10$ & $0.55 \%$ & $0.041 *$ \\
\hline Dysphagia & 43 & $2.22 \%$ & 10 & $0.62 \%$ & $<0.001 *$ \\
\hline Nervous & 47 & $2.42 \%$ & 74 & $4.55 \%$ & $<0.001 *$ \\
\hline Cardiac & 36 & $1.86 \%$ & 28 & $1.72 \%$ & 0.765 \\
\hline PVD & $\leq 10$ & $0.05 \%$ & $\leq 10$ & $0.12 \%$ & 0.463 \\
\hline Respiratory & 48 & $2.48 \%$ & 35 & $2.15 \%$ & 0.526 \\
\hline Digestive & 66 & $3.40 \%$ & 22 & $1.35 \%$ & $<0.001 *$ \\
\hline Urinary & 28 & $1.44 \%$ & 26 & $1.60 \%$ & 0.704 \\
\hline Device & 72 & $3.71 \%$ & 318 & $19.57 \%$ & $<0.001 *$ \\
\hline Shock & 11 & $0.57 \%$ & $\leq 10$ & $0.06 \%$ & $0.009 *$ \\
\hline Hematoma & 40 & $2.06 \%$ & 62 & $3.82 \%$ & $0.001 *$ \\
\hline Puncture & 33 & $1.70 \%$ & 54 & $3.32 \%$ & $0.001 *$ \\
\hline Infection & 73 & $3.76 \%$ & 92 & $5.66 \%$ & $0.007 *$ \\
\hline Anemia & 251 & $12.94 \%$ & 302 & $18.58 \%$ & $<0.001 *$ \\
\hline ARDS & 84 & $4.33 \%$ & 38 & $2.34 \%$ & $0.001 *$ \\
\hline $\mathrm{PE}$ & $\leq 10$ & $0.15 \%$ & 16 & $0.98 \%$ & $<0.001 *$ \\
\hline DVT & 27 & $1.39 \%$ & 45 & $2.77 \%$ & 0.003 * \\
\hline Shock - trauma & 16 & $0.83 \%$ & $\leq 10$ & $0.06 \%$ & $<0.001 *$ \\
\hline Infection - trauma & 73 & $3.76 \%$ & 92 & $5.66 \%$ & $0.007 *$ \\
\hline Any complication & 752 & $38.78 \%$ & 901 & $55.45 \%$ & $<0.001 *$ \\
\hline
\end{tabular}

$P V D$ peripheral vascular disease, $A R D S$ acute respiratory distress syndrome, $P E$ pulmonary embolism, $D V T$ deep vein thrombosis 
Table 2 Thoracolumbar spine surgery patients demographics, hospital characteristics, and complications

\begin{tabular}{|c|c|c|c|c|c|}
\hline Thoracolumbar surgery patients & \multicolumn{2}{|c|}{ Bariatric surgery $(N=740)$} & \multicolumn{2}{|c|}{ Morbidly obese $(N=740)$} & $P$ value \\
\hline Age & \multicolumn{2}{|c|}{$50.03 \pm 14.51$} & \multicolumn{2}{|c|}{$49.9 \pm 12.09$} & 0.851 \\
\hline Gender & \multicolumn{2}{|c|}{$493(67 \%)$} & \multicolumn{2}{|c|}{$486(65.7 \%)$} & 0.700 \\
\hline Invasiveness & \multicolumn{2}{|c|}{$6.89 \pm 2.49$} & \multicolumn{2}{|c|}{$6.96 \pm 2.39$} & 0.581 \\
\hline Hospital LOS & \multicolumn{2}{|c|}{$5.95 \pm 6.56$} & \multicolumn{2}{|c|}{$5.14 \pm 4.75$} & $0.007 *$ \\
\hline Complications & $N$ & Percentage & $N$ & Percentage & $P$ value \\
\hline Bowel issues & 91 & $12.30 \%$ & 89 & $12.03 \%$ & 0.87 \\
\hline Bladder issues & 12 & $1.62 \%$ & 19 & $2.57 \%$ & 0.20 \\
\hline Neurologic & $\leq 10$ & $0.95 \%$ & 12 & $1.62 \%$ & 0.25 \\
\hline Muscle weakness & $\leq 10$ & $0.27 \%$ & $\leq 10$ & $0.27 \%$ & 1.00 \\
\hline Nervous & 17 & $2.30 \%$ & 20 & $2.70 \%$ & 0.62 \\
\hline Cardiac & 17 & $2.30 \%$ & 11 & $1.49 \%$ & 0.25 \\
\hline PVD & $\leq 10$ & $0.14 \%$ & $\leq 10$ & $0.00 \%$ & 0.32 \\
\hline Respiratory & 19 & $2.57 \%$ & 19 & $2.57 \%$ & 1.00 \\
\hline Digestive & 37 & $5.00 \%$ & 11 & $1.49 \%$ & $<0.001 *$ \\
\hline Urinary & 14 & $1.89 \%$ & 11 & $1.49 \%$ & 0.55 \\
\hline Device & 45 & $6.08 \%$ & 172 & $23.24 \%$ & $<0.001 *$ \\
\hline Shock & $\leq 10$ & $0.54 \%$ & $\leq 10$ & $0.00 \%$ & $0.045^{*}$ \\
\hline Hematoma & 11 & $1.49 \%$ & 33 & $4.46 \%$ & $<0.001 *$ \\
\hline Puncture & 14 & $1.89 \%$ & 24 & $3.24 \%$ & 0.10 \\
\hline Infection & 33 & $4.46 \%$ & 40 & $5.41 \%$ & 0.40 \\
\hline Anemia & 150 & $20.27 \%$ & 135 & $18.24 \%$ & 0.32 \\
\hline ARDS & 31 & $4.19 \%$ & 15 & $2.03 \%$ & $0.02 *$ \\
\hline $\mathrm{PE}$ & $\leq 10$ & $0.41 \%$ & $\leq 10$ & $0.68 \%$ & 0.48 \\
\hline DVT & $\leq 10$ & $1.22 \%$ & 20 & $2.70 \%$ & $0.039 *$ \\
\hline Shock - trauma & $\leq 10$ & $0.81 \%$ & $\leq 10$ & $0.00 \%$ & $0.014 *$ \\
\hline Infection - trauma & 33 & $4.46 \%$ & 40 & $5.41 \%$ & 0.40 \\
\hline Any complication & 339 & $45.8 \%$ & 430 & $58.1 \%$ & $<0.001 *$ \\
\hline
\end{tabular}


Table 3 Cervical spine surgery patients demographics, hospital characteristics, and complications

\begin{tabular}{|c|c|c|c|c|c|}
\hline \multirow{2}{*}{$\begin{array}{l}\text { Cervical surgery patients } \\
\text { Age }\end{array}$} & \multicolumn{2}{|c|}{ Bariatric $(N=233)$} & \multicolumn{2}{|c|}{ Morbidly obese $(N=233)$} & \multirow{2}{*}{$\frac{P \text { value }}{0.767}$} \\
\hline & \multicolumn{2}{|c|}{$52.73 \pm 12.43$} & \multicolumn{2}{|c|}{$52.41 \pm 10.83$} & \\
\hline Gender ( $\%$ female) & \multicolumn{2}{|c|}{$123(52.8 \%)$} & \multicolumn{2}{|c|}{$127(54.5 \%)$} & 0.71 \\
\hline Invasiveness & \multicolumn{2}{|c|}{$6.89 \pm 2.36$} & \multicolumn{2}{|c|}{$6.85 \pm 2.37$} & 0.855 \\
\hline Hospital LOS & \multicolumn{2}{|c|}{$6.64 \pm 8.07$} & \multicolumn{2}{|c|}{$4.24 \pm 3.97$} & $<0.001 *$ \\
\hline Bowel issues & 17 & $7.30 \%$ & 32 & $13.73 \%$ & $0.02 *$ \\
\hline Bladder issues & $\leq 10$ & $2.15 \%$ & $\leq 10$ & $3.43 \%$ & 0.39 \\
\hline Neurologic & $\leq 10$ & $2.15 \%$ & 11 & $4.72 \%$ & 0.13 \\
\hline Muscle weakness & $\leq 10$ & $0.00 \%$ & $\leq 10$ & $0.86 \%$ & 0.16 \\
\hline Dysphagia & 11 & $4.72 \%$ & $\leq 10$ & $1.72 \%$ & 0.07 \\
\hline Nervous & $\leq 10$ & $0.86 \%$ & 15 & $6.44 \%$ & $<0.001 *$ \\
\hline Cardiac & $\leq 10$ & $1.29 \%$ & $\leq 10$ & $2.58 \%$ & 0.31 \\
\hline PVD & $\leq 10$ & $0.00 \%$ & $\leq 10$ & $0.00 \%$ & - \\
\hline Respiratory & $\leq 10$ & $3.00 \%$ & $\leq 10$ & $2.15 \%$ & 0.56 \\
\hline Digestive & $\leq 10$ & $1.72 \%$ & $\leq 10$ & $0.00 \%$ & $0.04 *$ \\
\hline Urinary & $\leq 10$ & $1.29 \%$ & $\leq 10$ & $0.43 \%$ & 0.32 \\
\hline Device & $\leq 10$ & $3.00 \%$ & 42 & $18.03 \%$ & $<0.001 *$ \\
\hline Shock & $\leq 10$ & $0.43 \%$ & $\leq 10$ & $0.00 \%$ & 0.32 \\
\hline Hematoma & $\leq 10$ & $2.58 \%$ & $\leq 10$ & $1.72 \%$ & 0.52 \\
\hline Puncture & $\leq 10$ & $1.29 \%$ & $\leq 10$ & $2.58 \%$ & 0.32 \\
\hline Infection & $\leq 10$ & $2.58 \%$ & $\leq 10$ & $1.72 \%$ & 0.52 \\
\hline Anemia & 22 & $9.44 \%$ & 14 & $6.01 \%$ & 0.17 \\
\hline ARDS & 15 & $6.44 \%$ & 11 & $4.72 \%$ & 0.42 \\
\hline $\mathrm{PE}$ & $\leq 10$ & $0.00 \%$ & $\leq 10$ & $0.86 \%$ & 0.16 \\
\hline DVT & $\leq 10$ & $1.29 \%$ & $\leq 10$ & $1.29 \%$ & 1.00 \\
\hline Shock - trauma & $\leq 10$ & $1.29 \%$ & $\leq 10$ & $0.00 \%$ & 0.08 \\
\hline Infection - trauma & $\leq 10$ & $2.58 \%$ & $\leq 10$ & $1.72 \%$ & 0.52 \\
\hline Any complication & 79 & $33.9 \%$ & 187 & $80.3 \%$ & $<0.001 *$ \\
\hline
\end{tabular}

